\section{Identifying Foreign Material Contamination in Food and Food Ingredients}

\author{
V. L. St. Jeor, A. Lape, A. R. Muroski, C. McGuire, \\ J.S. Kruger, and D. L. Elmore \\ Cargill Global Food Technology Group, Cargill Incorporated, \\ Excelsior, MN \\ Var_StJeor@cargill.com
}

\section{Introduction}

Between the Biological and Materials sciences resides the food industry, where both biological and materials methods and techniques are employed for both production and analysis. Often referred to as "Black Specs," one of the biggest concerns for the food industry is contamination of the food, or its additives and ingredients, with foreign material (FM). Many of our customers seek assistance in solving their FM issues, which may require multiple instrumentation, several methods, and considerable open communication with the customer before a satisfactory result can be achieved. An additional level of difficulty is often present when we learn that the FM is so small we can barely detect it with the unaided eye. Particularly difficult is what might be referred to as "chemical contamination." We present here in example (and in the upcoming Microscopy and Microanalysis meeting in Albuquerque this August), several of the methods employed in assisting our customers with FM issues.

Though it is not usually necessary to learn everything about the FM of interest during the investigation, it is usually necessary to learn key pieces of information that can be used, either by us, or the customer, to solve the problem and resume production. Remaining in frequent contact with the customer helps for three reasons: 1 ) We often need to know more about the submitted samples than initially shared by the customer. 2) Intellectual property (IP) issues can become a concern for the customer when sharing information with their supplier. This is best worked out over time, building levels of trust and confidence. Without trust and communication, the associated costs to solve the problem can increase through wasted efforts and false leads. 3) Once enough information has been collected to solve the problem, from the customer's perspective, the customer can help determine when the investigative process can stop. This approach produces a financial savings, and benefits both parties.

\section{Methods}

In our attempt to share some of our experience, we must make note: there is a nearly endless list of possible FM. Each type can present its own set of challenges. We offer examples here of only a few. Therefore, experience and ingenuity become critical in choosing, or (more likely) developing, methods needed to answer the requests. Some of the questions we may need to answer include: 1) what is the material, and/or its elemental or chemical composition? 2) Where might it originate or what was the parent object? 3) Is there more than one material involved? 4) How did it get into the food? 5) Does the FM match a control material? 6) How was the FM formed? We will examine elemental and chemical content, morphology, color, deformation patterns, etc. Examples of methodology often employed include: light or electron microscopy (LM or SEM), x-ray-based energy dispersive spectroscopy (EDS), $\mathrm{X}$-ray fluorescence (XRF), Fourier-transform infrared spectroscopy
(FT-IR), Raman spectroscopy, Fluorimetry, Inductively Coupled Plasma Emission Spectrometry (ICPES), and various forms of Mass Spectrometry, Liquid Chromatography, Gas Chromatography with Olfactory detection (GCO), etc., with some of the latter instruments being combined to work together.

\section{Example Results}

With such forensics, whether the FM is something simple like a human hair or a nylon fiber, or something more challenging like a dextrin contaminated with some other dextrin, it is our job to identify the FM and in some cases, propose possible methods for how the contaminant might have found itself in the food or ingredient. In some instances, one finds something that is not supposed to be there. In other cases, the only way we know something is wrong is that the food is not acting correctly. Perhaps it separates, shrinks, waters-off, clumps, or displays the wrong color or the wrong viscosity. Therefore, part of the job of forensics can include diagnosis.

Generally, the customer makes some assumptions, which cause them to draw the conclusion that it must be something they buy from Cargill. Surprisingly, that conclusion is generally wrong more often than not. We then usually hear something like, "Well, if it isn't your ingredient, what is it?" That leaves us with the remaining ingredients in their product (which the customer may or may not share with us). To the customer's surprise, we often have the capacity to determine what those other ingredients are and, even though we have little experience with these other ingredients, we must often identify not only the faulty ingredient, but also what we think is wrong. That can place us in the awkward position of carefully playing the diplomat. However, let's start with something relatively simple.

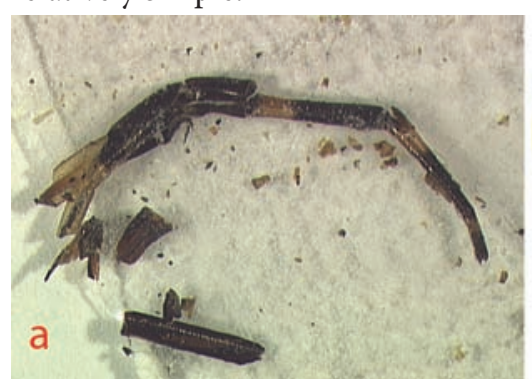

Fig. 1a. An LM image of a portion of a dehydrated spider's leg.

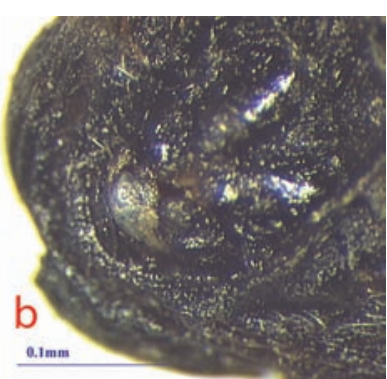

Fig. 1b. The posterior region of the spider's abdomen, in ventral view, showing the spinnerets.
Morphology is often used to identify certain types of FM. Figure 1a-b indicates an example of what a customer described as "bug parts," with instructions to "...please identify and determine contamination source." That is a tall order. The number of collected body parts gave the customer the idea that the FM represented several "insects." In such cases, it helps to have a little entomology background, or a local "expert" to which images can be taken. We were able to piece the small collection of parts back together roughly and, although the cephalothorax was missing (the combined head/ thorax structure), the abdomen and its spinnerets Figure $\mathbf{1 b}$, and the number of legs were used to determine the creature was a single, large, highly desiccated spider; one with which we were not familiar. The customer used leg markings (Figure 1a) observed in our reported images, to determine the spider is common to their region of the country, and is often observed in their plant.

Metal contamination, often small flecks collected by the customer's filters, magnets, etc., usually require multiple methods of 


\section{New Plasma Trimming ${ }^{\mathrm{TM}}$ Tools}

\section{Thins and Removes Amorphous Ion Damage}
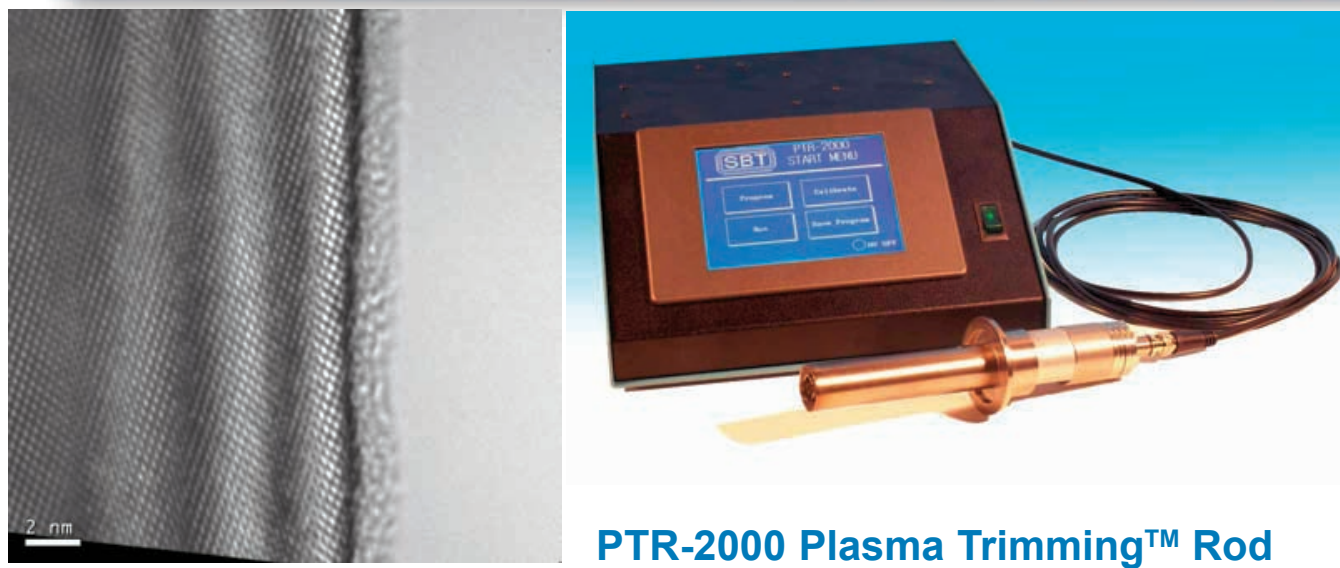

PTR-2000 Plasma Trimming ${ }^{\mathrm{TM}}$ Rod

Easy to use.

$>$ Compatible with all FIB instruments.

$>$ Compatible with different gases and gas mixtures.

$>0$ to -1000 Volts (PCPT)

$>$ Programmable 0 to -500 Volts (PTR-2000).

$>$ Uses modified Fortress ${ }^{\mathrm{TM}}$ holders.

$>$ Compatible with SampleSaver ${ }^{\mathrm{TM}}$ storage containers.

$>$ Does not affect plasma cleaning capabilities.

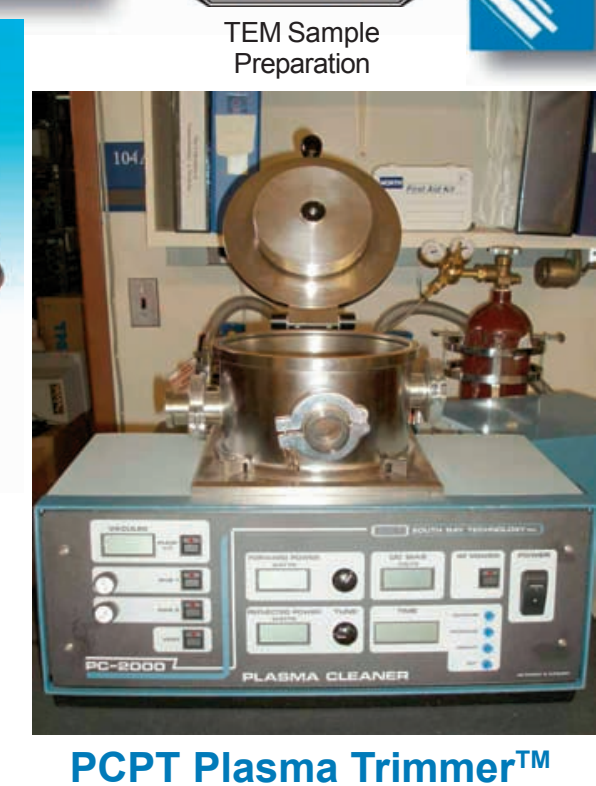

South Bay Technology, Inc. has developed two Plasma Trimming ${ }^{\mathrm{TM}}$ tools for cleaning the amorphous damage from FIB-prepared, ion milled, or Tripod polished TEM samples with low energy ions. Five modified Fortress ${ }^{\mathrm{TM}}$ holders and the SBT SS-100 SampleSaver ${ }^{\mathrm{TM}}$ with FIB rack are included to minimize re-oxidation of the samples until they are ready to be imaged.

\section{New UVO Cleaner ${ }^{\circledR}$ Station*}

\section{Stores and Cleans TEM Sample Holders in Air!}

${ }^{*}$ UVO-Cleaner ${ }^{\circledR}$ is a registered trademark of Jelight Company Inc.

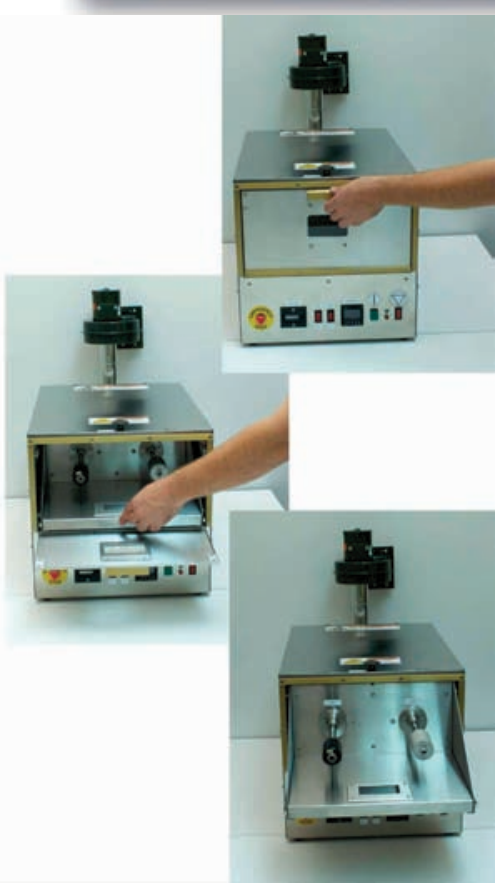

The UVO Cleaner ${ }^{\circledR}$ Station is designed to store multiple TEM specimen holders while periodically cleaning them using specially designed, spiral UV lamps using the UVO cleaning method. Each specimen holder is securely held in an adapter that is specific to its manufacturer's design. This adapter is mounted on the rear panel of a sliding drawer. When the drawer is closed, the rods are centered within the spiral lamps while keeping the rods in a Available Models: dust-free container. When on, the spiral lamps surround the rod within a few millimeters and completely bathe the rod in UV light generating active oxygen species for cleaning.

UVO-CS2-2: 2-position, 2 active lamps

UVO-CS4-2: 4-position, 2 active lamps

UVO-CS4-3: 4-position, 3 active lamps

UVO-CS4-4 4-position, 4 active lamps E 을 


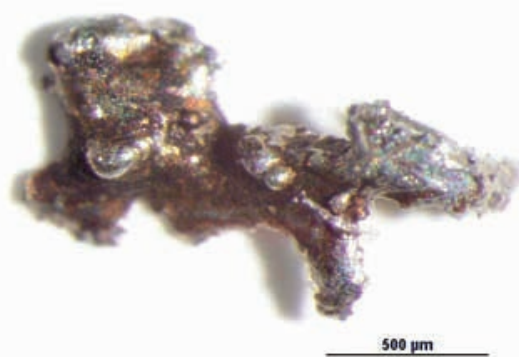

Fig. 2. An LM image of a small piece of stainless steel weld splatter.

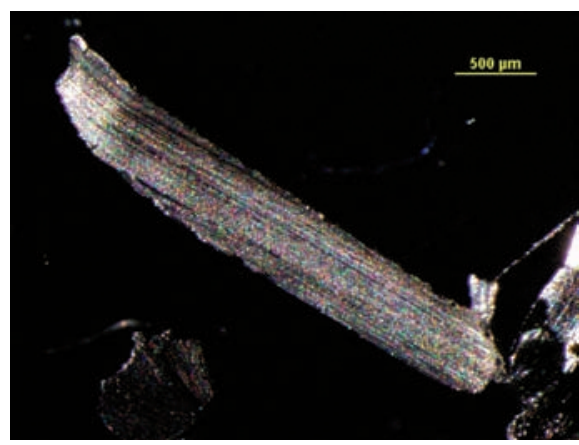

Fig. 3. An LM image of a small filing of stainless steel displaying tool marks.

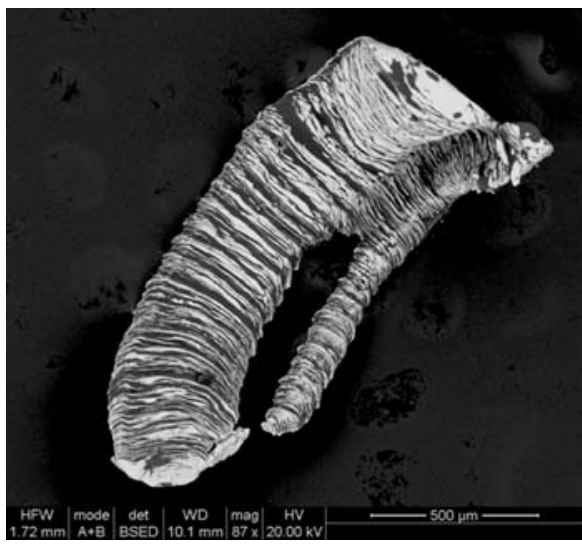

Fig. 4. A BSE-SEM image of a stainless steel fragment with a contaminating partial coating. The fragment has kinked and buckled many times to produce the corrugated appearance. identification. Figures $\mathbf{2 - 4}$, indicate three differing morphologies of a common form of contamination in the food industry: stainless steel. EDS can quickly identify the stainless steel, but morphology can often tell us, how the FM was formed and possibly how it came to be in the sample. Figure 2 indicates stainless steel that had been in a molten state. Note the rounded and bubbly appearance of some of the protruding domes. We identified this as stainless weld splatter. Figure 3 indicates a thin strip of stainless displaying distinctive linear marking, sometimes referred to as tool marks. This morphology indicates that the strip was shaved from the parent material in some fashion, and can be an indicator of things like abnormal ware, or an out-of-adjustment belt or failing bearing, which can cause parts to rub together. A discoloration in the metal, such as bluing, can indicate high heat due to friction combined with high speed.

Figure 4 is an SEM image, using backscatter electron (BSE) mode, of a piece of stainless steel. The BSE detector is set in compositional mode, meaning it can detect compositional differences between differing materials. We note two features: 1) The wrinkly appearance of the metal and, 2) a partial coating of some other substance, which is resting in the valleys between wrinkles, and which displays a darker appearance in BSE mode than the bright-appearing stainless (compositional contrast). The darker substance (as seen in BSE mode) is not evident with light microscopy. The wrinkly morphology in the stainless steel is the result of the gouging process that produced the metal fleck. As the fleck was being removed, it would compress, buckle, kink, and fold back and forth on itself repeatedly, in essence creating an accordion-like appearance. Thus, the resulting metal fleck would be considerably shorter than its associated gouge mark left in the parent material. The dark coating material (based upon EDS analysis) is a carbon-based material that the customer determined was the food material being processed.

Figure 5a-b is an example involving multiple materials. Basically, the material consists of very small flakes of metal sometimes displaying a white coating. EDS, elemental mapping and X-ray fluorescence identifies the metal as an alloy steel (possibly a manganese steel), and the coating as two chemically differing layers, possibly paint or a sprayed liner. Figure $\mathbf{6 a}-\mathbf{b}$ are the EDS spectra showing the metal and coating and the metal alone (respectively). A close examination of the EDS X-ray maps and their associated low resolution, EDS system-generated SEM images indicate that the first particle (7a-b) displays remnants of a heavy coating (both coating layers being intact), while a third particle (7c-d) displays bits and pieces of the coating (the under-layer only). (Note: Figures $7 \mathbf{a}$ and $5 \mathbf{a}$ are the same particle using differing microscopes.)

Both coating layers are high in Titanium, but one contains the extras Silicon, Magnesium and other elements. For the monolayer maps, Magnesium, like Titanium, is everywhere the coating exists. For the bi-layer maps (7a-b), with upper layer in place, Magnesium is apparent only in local clusters at the edges and where the top layer is thin or missing, while Titanium is present everywhere in both layers. Therefore, we can identify two coating layers. Morphology of the metal itself is consistent with flaking due to impact fatigue. This was sufficient information for the customer to locate the source within their processes.
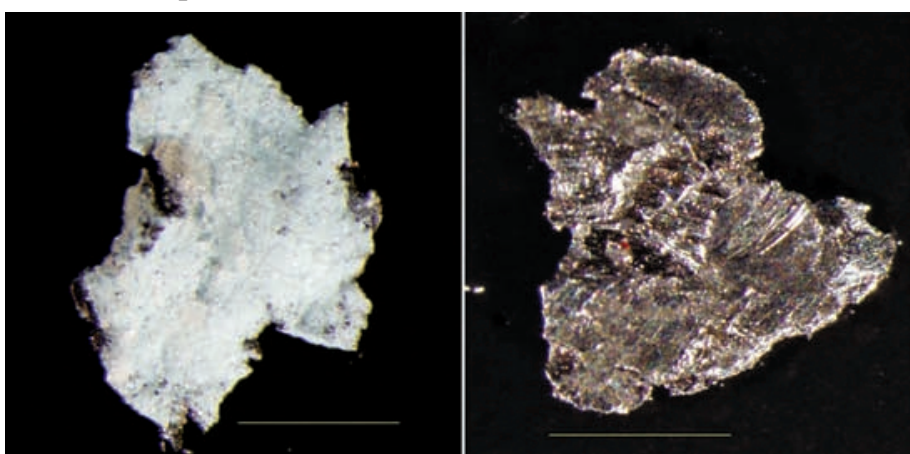

Fig. 5a-b. Two metal flakes from the same sample, one displaying $a$ white coating ( $5 a)$, the other (5b) without.

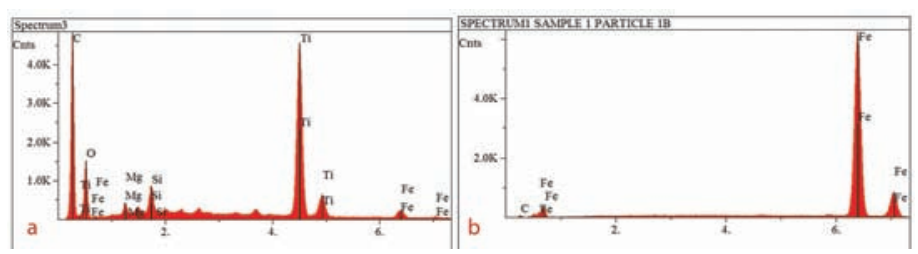

Fig. 6a-b. EDS spectra displaying elemental content. $6 a$ shows the metal with the coating material, $6 b$ shows the metal only.

We are often asked to determine a specific source material for a particular FM, and to assist a customer in tracing down the specific source within processing. For polymers, Raman or FT-IR might be the best available option, but for metals, X-ray fluorescence (XRF) is often the best way to determine elemental fingerprint because of its ability to detect minor components. XRF uses an X-ray source to generate characteristic sample $\mathrm{x}$-rays rather than the electron probe of the SEM (hence the name $x$-ray fluorescence). Having our x-ray source on the SEM, we simply use the same x-ray detector used for EDS, but with the SEM accelerating voltage turned down to 1 or $2 \mathrm{KV}$, just enough to see the sample well, but we have little or no input from the electron beam for $\mathrm{x}$-ray generation. Our $\mathrm{x}$-ray source 



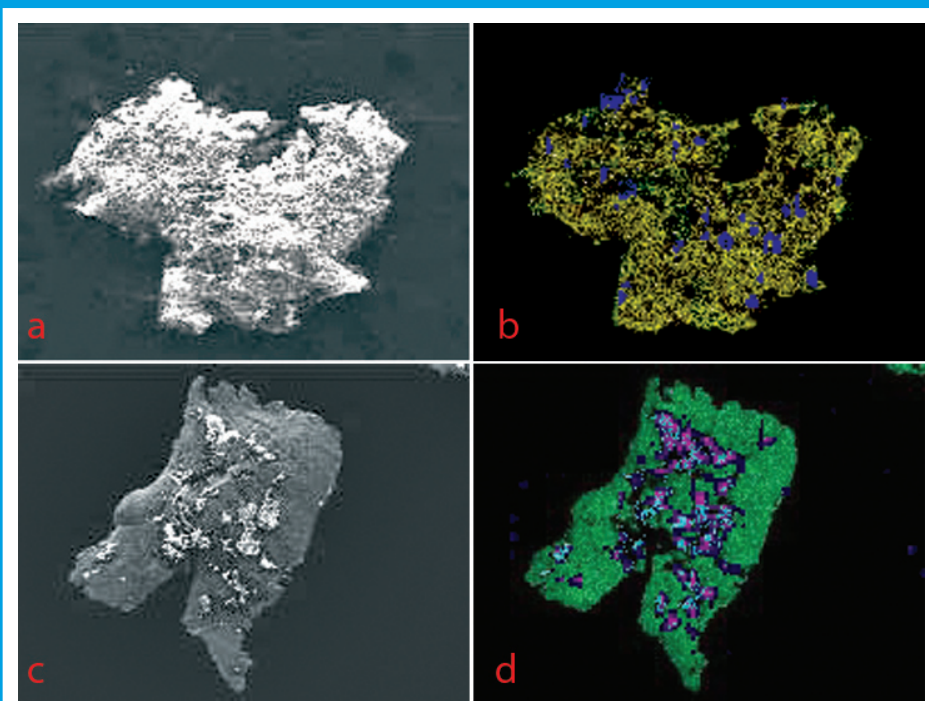

Fig. 7a-d. Low resolution SEM images of two particles ( $7 a$ \& c), with their associated EDS-generated elemental maps ( $7 b \& d) .7 b$ displays $T i$ as yellow dots and $\mathrm{Mg}$ as clusters of blue dots. $7 d$ displays Fe as green, $\mathrm{Ti}$ as light blue and $\mathrm{Mg}$ as pink.

generates $\mathrm{x}$-rays at $\sim 50 \mathrm{KV}$ and produces a focused $\mathrm{x}$-ray beam of $\sim 50$ micrometers in diameter.

Figure 8a-b indicates an example comparison of XRF data for an unknown Stainless with two known Stainless prospects supplied by the customer. Only one of the two known samples is consistent with the unknown. The main differences lie in the copper peaks, and some minor elemental differences noted out past $11 \mathrm{KV}$. The unknown is consistent with the known sample containing the copper.

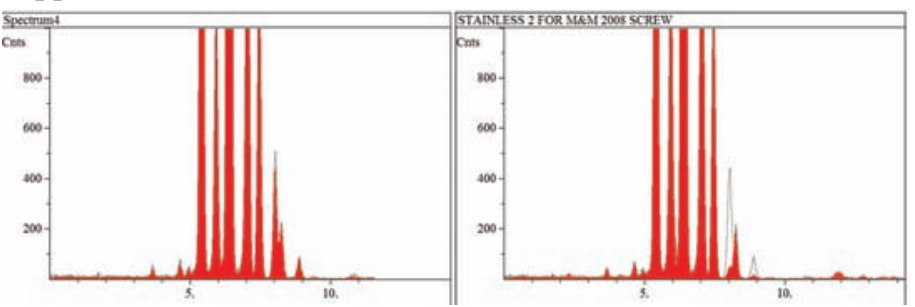

Fig. 8a-b. 8a shows overlay XRF spectra of a known stainless steel (red) and an unknown (trace). $8 b$ shows an overlay XRF spectra of a differing known stainless steel (red) and the same unknown (trace). $8 \mathrm{a}$ indicates that its known and the unknown are consistent. 86 indicates no consistency.

The presence of copper is unusual in that although some stainless steels contain some copper, most do not. We suspect the relatively small quantity of copper observed to be incidentally present within the stainless steel. However, it is equally present in the unknown making the unknown sample a virtually identical match to the copper-containing known sample.

Another way to analyze for elemental content is through Inductively Coupled Plasma Emission Spectrometry (ICPES), which can provide elemental data about certain types of materials with greater efficiency than EDS. In one case, the customer was concerned about a very slight discoloration in the product, referred to as "yellowing," when compared to a control. Using sample digestion and an elemental spectrum scan method, semi-quantitative results (based upon single point method of a known reference) indicated elevated levels of sulfur, sodium, silicon, potassium, calcium and magnesium when compared to that of the control. Particularly high concentrations of sulfur and magnesium were documented. Further ICPES testing of selected samples, which traced the product through its

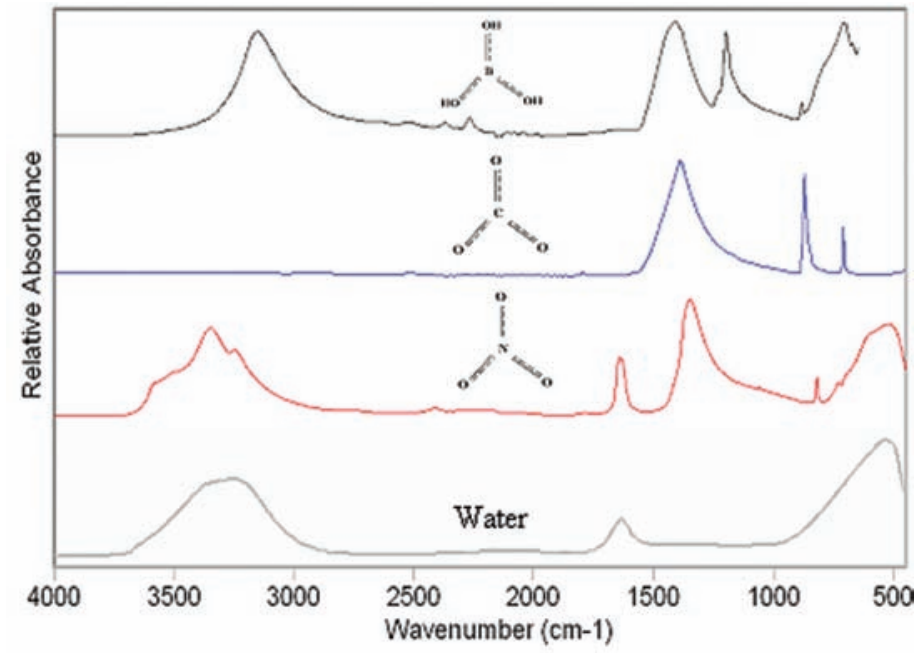

Fig. 9. FT-IR ATR spectra showing the structurally similar compounds boric acid, carbonate and nitrate, compared to the spectrum for water. In this case, water can interfere with the other three spectra making sure chemical identification difficult to achieve, with out EDS.

stages of processing, allowed us to determine where in processing the discoloration occurred.

Food matrices can be particularly difficult for chemical FM analysis. Many foods carry a fair amount of water, carbohydrate, and/or lipid, which can mask other components in FT-IR ATR spectra. Confident characterization of this FM usually requires a combination of molecular, elemental, and morphological information for sufficient specificity in complicated systems. We have combined, with particular effectiveness, molecular data from FT-IR, and elemental information from EDS. A good example of this synergy is differentiation among the structurally similar polar compounds boric acid, carbonate, and nitrate (Figure 9), where water can mask important chemical structure in FT-IR spectra. It is necessary to know both the chemical structure, obtained by FT-IR analysis, and the elemental composition obtained by energy-dispersive $\mathrm{x}$-ray spectroscopy (or perhaps ICPES) before we can clarify the IR data in such cases.

Our laboratory deals with flocculation and sediment issues in many types of beverages. These flocs and sediments are usually

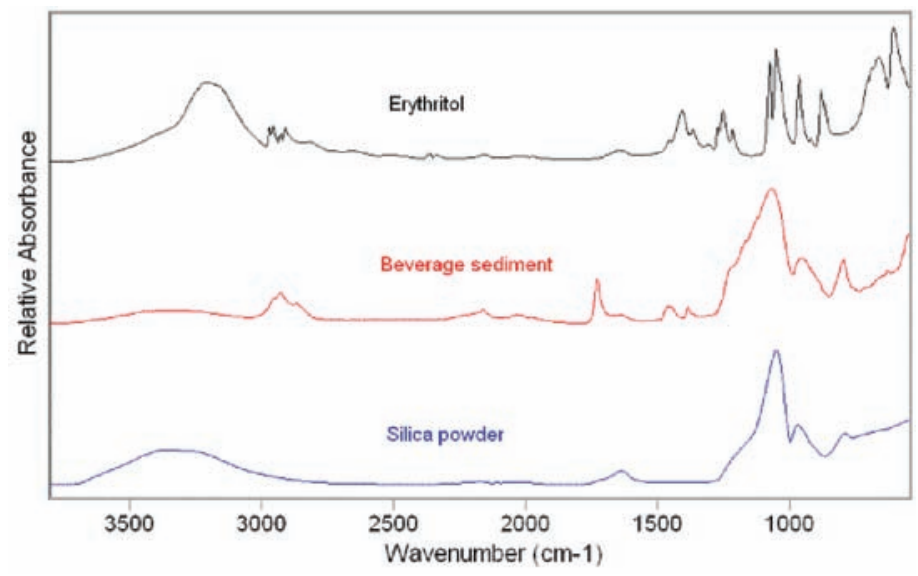

Fig. 10. FT-IR diamond ATR spectra of erythritol (top), typical beverage sediment (middle), and silica powder (bottom). The sediment spectrum is clearly not similar to that of erythritol, and has main features similar to those of the silica spectrum. Additional $\mathrm{C}-\mathrm{H}$ and carbonyl bands reveal the presence of fatty ester as a second main component of the sediment. 


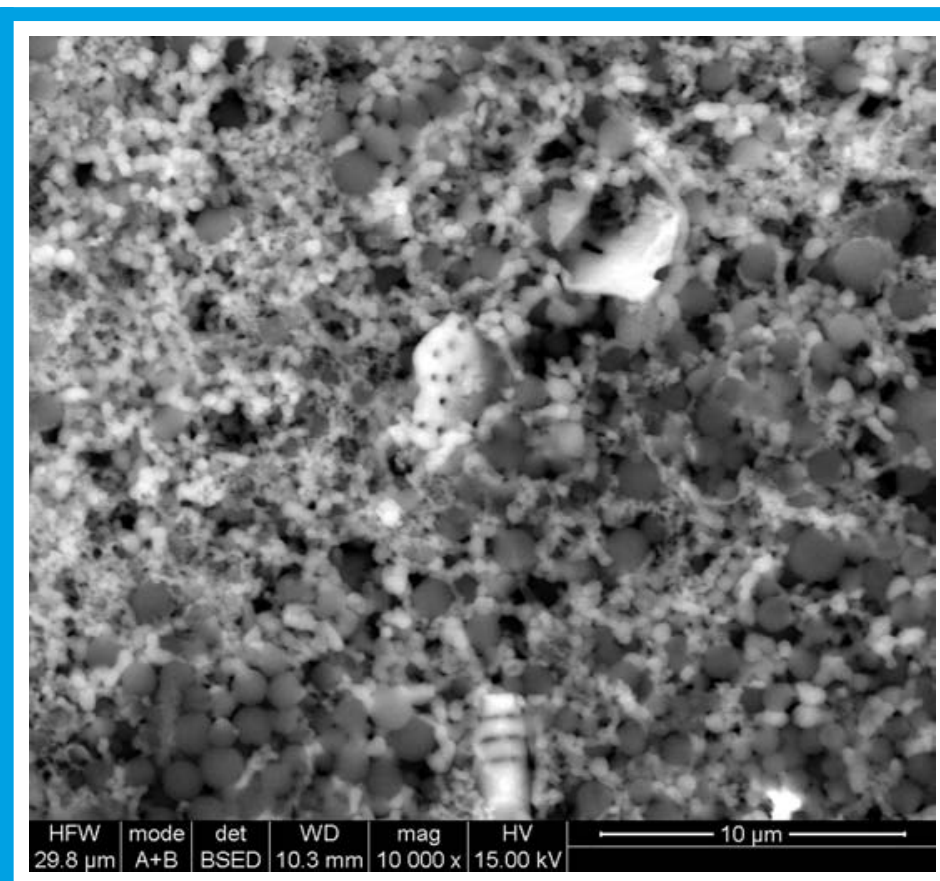

Fig. 11. Scanning electron microscope-BSE image (in low-vacuum mode) of beverage sediment, showing larger, darker, lipid-like spheres embedded in a matrix of amorphous and diatomaceous silica.

a combination of things, not limited to the beverage ingredients. In most instances, the flocs and sediments contain actual foreign material, like plastic fibers, cellulosic fibers, and nondescript particulates. Moreover, the beverage ingredients are typically sourced from several different suppliers, and the flavor packages, in particular, are often proprietary. When trying to identify problems in these beverages, therefore, we often are at a loss for ingredient references. In such situations, we must rely on a combination of complimentary analytical techniques for confident assessments of the offending materials, since a single technique usually provides not enough information from multi-component mixtures.

Perhaps the most critical aspect of properly identifying beverage FM is its isolation from the bulk. Most of the flocs and sediments we have seen are very delicate, and are present in tiny amounts. Simply inverting the products' retail bottles is often sufficient to completely disperse the FM into the bulk. Collecting and washing enough FM for replicate analyses is painstaking work, often with a micro-pipette while viewing the sample through a stereoscope. The stereoscope is invaluable for a first-look at the FM, to assess morphology, color, size, etc. This initial examination allows us to determine how to proceed with cleaning and spectroscopic analyses, and serves as a reference for the FM's "native" state.

We generally begin our FM measurements with Fouriertransform infrared spectroscopy (FT-IR). We use an attenuated total reflection (ATR) sampling device, because it allows minimal sample preparation; indeed, most times we simply can transfer the analyte suspended in solvent directly to the ATR sampling surface.

A good example of our beverage materials ID work is a recent problem with sediment in a customer's new low-calorie vitaminenriched product, in which a main ingredient is erythritol, one of Cargill's natural sweeteners. Of course, the customer implicated erythritol, since the customer has relatively little experience using this ingredient in its formulations.

The sediment was difficult to isolate, as it requires weeks to form, and tends to easily re-disperse. By combining samples from

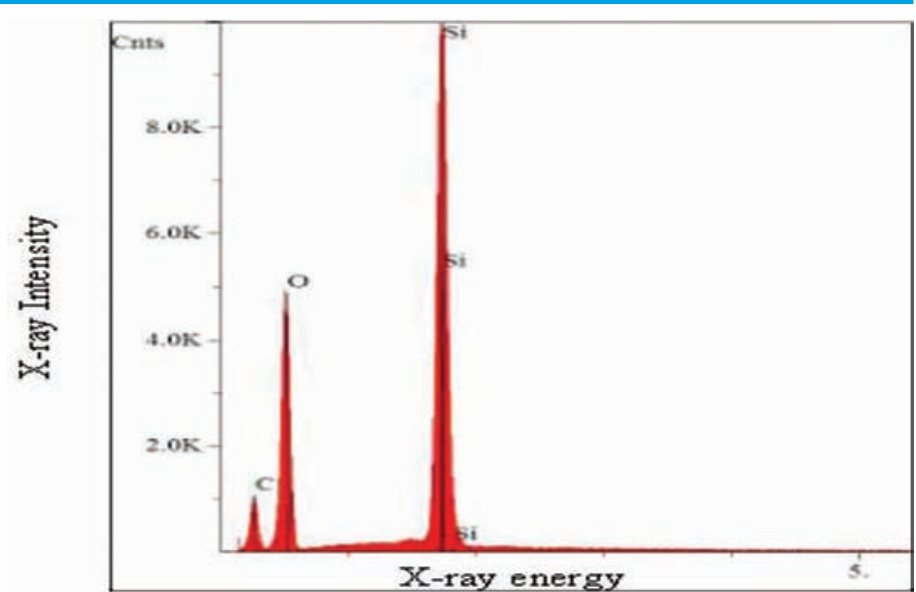

Fig. 12. Energy-dispersive X-ray spectrum of the bulk sediment, indicating strong silicon and oxygen signals from silica. The carbon and a small amount of the oxygen signal are due to ester.

several cases of product, we were able to isolate and wash enough sediment for examination by FT-IR, SEM, and EDS.

Figure 10 compares a typical FT-IR ATR spectrum collected from the sediment with a spectrum of erythritol. These profiles are very different, indicating the sediment contains no significant amount of erythritol. Instead, the sediment spectrum matches well with a spectrum of amorphous silica. The sediment's profile also contains bands attributable to various fatty esters in the product's formulation. These FT-IR data reveal the chemical identities of two main sediment components; however, they do not show how these components are interacting to cause the problem.

Figure 11 is an SEM-BSE image of the sediment, with the SEM in low vacuum mode. From this image, we can see what appear to be darker, lipid-like spheres embedded in a matrix of smaller, brighter spheres, including some diatoms. These larger, darker spheres are likely the fatty esters indicated by the IR measurements, and the diatomaceous material is clearly silica. EDX spectra collected from the sediment (Figure 12) contain strong silicon and oxygen signals, supporting the idea that the sediment is mostly silica. In fact, the IR data show the silica in the sediment is mainly amorphous, rather than crystalline, explaining the smaller, brighter spheres. This synergy of chemical information from FT-IR and morphology/ elemental insight from SEM allowed us to conclude the sediment seems to form by gradual agglomeration of fatty esters with small silica particles.

Upon subsequent examination of some of the product's ingredients, we determined amorphous silica is present in excessive amounts in one of the key ingredients. In the end, our broad technical approach not only exonerated Cargill's product, but also showed the customer and its other suppliers how to solve their problem, which facilitated their new product launch.

\section{Conclusions:}

1. Try to understand exactly what the problem is, which can, at times, differ from what the customer is requesting.

2. Try to determine the best course of action and the optimum instrument of choice at the start. Ingenuity can be the best instrument in your arsenal.

3. Remain open-minded at all stages of the investigation.

4. Maintain frequent communication with the customer.

5. Don't isolate yourself, but rather work closely with the customer.

6. Respect customer IP and trade secrets. 